Case Report

\title{
Major Cognitive Changes and Micrographia following Globus Pallidus Infarct
}

\author{
Sarah Nelson, ${ }^{1}$ Hassanain Toma, ${ }^{2}$ Haley LaMonica, ${ }^{3}$ and Tinatin Chabrashvili ${ }^{3}$ \\ ${ }^{1}$ Massachusetts General Hospital, 55 Fruit Street, Boston, MA 02114, USA \\ ${ }^{2}$ Royal Columbian Hospital, 330 E Columbia Street, New Westminster, BC, Canada V3L 3W7 \\ ${ }^{3}$ Tufts Medical Center, 800 Washington Street, Boston, MA 02111, USA \\ Correspondence should be addressed to Sarah Nelson; senelson13@gmail.com
}

Received 25 August 2014; Revised 21 October 2014; Accepted 21 October 2014; Published 12 November 2014

Academic Editor: Alfonso Fasano

Copyright (C) 2014 Sarah Nelson et al. This is an open access article distributed under the Creative Commons Attribution License, which permits unrestricted use, distribution, and reproduction in any medium, provided the original work is properly cited.

Importance. Globus pallidus (GP) lesions are well known to cause motor deficits but are less commonly-and perhaps not conclusively-associated with cognitive problems. Observations. We present a 45-year-old male with no significant neurological or psychological problems who after suffering a GP infarct was subsequently found to have substantial cognitive problems and micrographia. Formal neuropsychological testing was not possible due to lack of patient follow-up. Conclusions and Relevance. Despite the conflicting literature on the association of GP lesions and cognitive deficits, our patient demonstrated significant neuropsychological changes following his stroke. In addition, evidence of micrographia likely adds to the literature on the localization of this finding. Our case thus suggests that neuropsychological testing may be beneficial after GP strokes.

\section{Case Report}

A 45-year-old right-handed male with a history of hypertension, diabetes mellitus, and depression-but no other known cognitive issues-presented in November 2013 with altered mental status. He had been found stuck in a basement alcove for an unknown period of time prior to being brought to the emergency room by emergency medical services, and the patient was unable to provide an accurate timeline of events leading up to his presentation. On neurologic examination in the emergency room, he was slow to respond to questions and did not know the correct date. Right-sided weakness but no Parkinsonian features or obvious changes in tone were noted. Magnetic resonance imaging (MRI) of the brain demonstrated a left acute to subacute GP infarct and an old right GP lacunar infarct (Figure 1). Magnetic resonance angiogram of the head and neck was normal. Basic laboratories on admission were generally unremarkable except for glucose $438 \mathrm{mg} / \mathrm{dL}$, creatinine $1.45 \mathrm{mg} / \mathrm{dL}$, and a white blood cell count of $13 \mathrm{~K} / \mathrm{uL}$; all of these values normalized during his hospital stay. A serum toxicology screen on admission was unremarkable. Low-density lipoprotein was $160 \mathrm{mg} / \mathrm{dL}$ and hemoglobin Alc was 12.0\%. Transthoracic echocardiogram showed a normal ejection fraction and no interatrial shunt. Telemetry showed no signs of arrhythmia.

The patient was started on aspirin and atorvastatin as well as medications to control his blood pressure and diabetes. On discharge two days later, he exhibited mild right-sided weakness and slight problems in orientation; he knew that he was in the hospital but did not know its name and could not state the exact date in November 2013.

However, five days later the patient again presented with altered mental status. He was oriented to self only, produced only one-word answers with considerable prompting, and appeared abulic. Overall, his family felt he was a completely different person than prior to his stroke. Repeat MRI of the brain showed interval evolution of the left BG infarct but no other new findings. No other causes for his exam findings (including seizure, infection, or toxic-metabolic etiologies) were discovered. He was discharged in early December 2013 on similar medications as on his prior admission.

The patient did not follow up for formal neuropsychological tests, but he had undergone bedside testing during the latter half of his second hospital stay. Deficits in the 


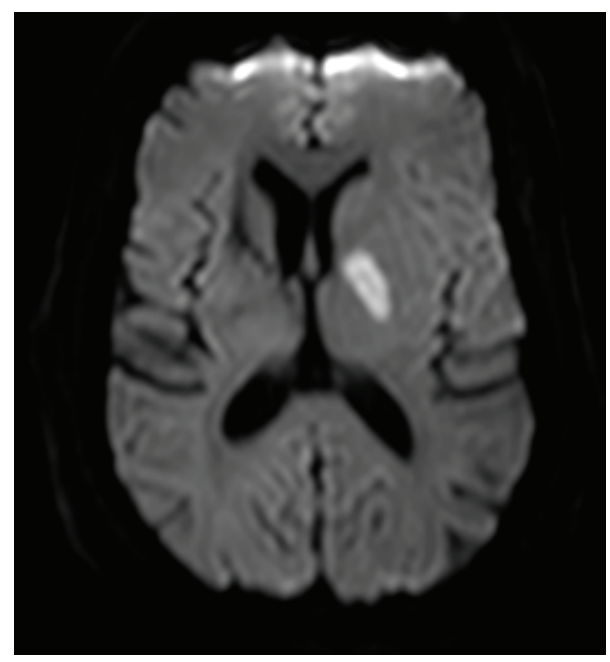

FIGURE 1: Axial MRI of the brain. Diffusion weighted imaging sequence showing acute left globus pallidus infarct.

TAble 1: Montreal Cognitive Assessment cognitive domains (derived from [7]).

\begin{tabular}{lcc}
\hline Cognitive domain & $\begin{array}{c}\text { Patient's } \\
\text { score }\end{array}$ & $\begin{array}{c}\text { Normal } \\
\text { score }\end{array}$ \\
\hline Short-term memory & 0 & 5 \\
Visuospatial ability & 1 & 4 \\
Executive function $^{*}$ & 0 & 4 \\
Attention/concentration/working memory $^{*}$ & 2 & 6 \\
Language $^{*}$ & 4 & 6 \\
Orientation $_{\text {Total }}$ & 5 & 6 \\
\hline
\end{tabular}

* Because the phonemic fluency task (1 point) tests both executive function and language, the total number of possible points in this table is 31 .

Montreal Cognitive Assessment were present in all domains but were most prominent in short-term and working memory, visuospatial ability, executive function, attention, and concentration (Table 1). He perseverated in the alternate triangles and squares sequencing task. Expressive aphasia was evident while describing the Boston Diagnostic Aphasia Examination's Cookie Theft picture. Micrographia, primarily with his right hand, was also present (Figure 2).

In a follow-up visit with his primary care physician 3 days after discharge from the second hospital admission, he was noted to be oriented to person and date, but not to location. The patient displayed language and comprehension deficits: he was "unable to follow commands to fully assess strength and rarely speaks in full sentences but can answer simple sentences with great effort." However, more rigorous testing of other aspects of his cognition was not performed.

\section{Discussion}

GP lesions are typically associated with motor deficits, including Parkinsonism, tremor, and dystonia $[1,2]$. However, somewhat controversially and undoubtedly less commonly,

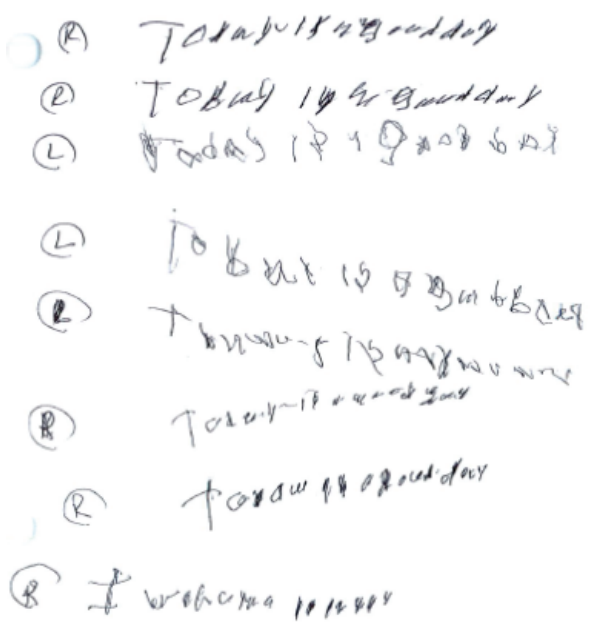

FIGURE 2: Handwriting samples. Micrographia was primarily demonstrated in the patient's right hand (designated " $R$ ") as compared to his left hand (designated "L").

these lesions may also be associated with cognitive disorders and micrographia.

Many of the studies evaluating cognitive problems following GP lesions derive from the Parkinson's disease (PD) literature. Following pallidotomy or other surgical lesioning of the GP in PD, various cognitive changes occurred in two studies including abulia, mental automatism, depression, and deficits in attention, naming, encoding, retrieval, and motor learning and speed $[3,4]$. In one study, globus pallidus interna (GPi) lesion location along anteromedial to posterolateral axis was associated with both cognitive and motor postsurgical outcome [5]. In a review, 5 of 16 studies on GPideep brain stimulation (DBS) reported cognitive changes such as mania, hypomania, suicide, and improved depression. Eleven out of 25 studies regarding pallidotomy described behavior problems including depression, transient confusion, hallucinations, psychotic depression, and paranoia [6].

Specific to the stroke literature, cognitive effects after GP infarction include executive function problems, verbal memory problems, abulia, loss of emotional expression, reduction of spontaneous thought content, disinhibition, and obsessive-compulsive disorders $[8,9]$. Small subcortical lesions involving the thalamus, caudate, and GP appear to disrupt cortico-subcortical circuits, resulting in cognitive dysfunction [10]. In yet other literature on GP lesions due to other reasons, worsening general cognition, immediate recall problems, and improvement in executive function were demonstrated following GPi lesions [2, 11, 12].

Conversely, other literature has shown no association between the GP and cognition. For example, several studies showed no significant cognitive changes after pallidal DBS in PD patients [13-16]. Similarly, there were generally no neuropsychological changes after pallidotomy in many studies [17-21]. A meta-analysis of 240 patients with caudate, putamen, and GP lesions due to various causes revealed that motor deficits were frequently associated with lentiform 
nucleus lesions. Few behavioral abnormalities were associated with GP lesions and only with bilateral lesions [1].

Furthermore, our patient displayed evidence of micrographia. Small handwriting is a signature feature of Parkinson's disease, which is caused by loss of dopaminergic neurons in the substantia nigra pars compacta. As shown in the small body of literature on micrographia associated with stroke, localizations of this symptom following stroke have included frontal lesions (e.g., anterior cerebral artery infarctions), left thalamomesencephalic region, and BG [2228]. One study demonstrated that micrographia could in fact indicate the presence of a stroke [27]. Another article's authors emphasized that, up until their article, the left BG-thalamic region was involved in all reported cases of micrographia after subcortical infarction [28]. Most relevant to our patient, four cases of micrographia were due to strokes in the lenticular nucleus [29]. In addition, other GP lesions have been found to cause micrographia, including GPiDBS, a finding that was thought to be a manifestation of hypokinesia due to disturbance in pallido-thalamo-cortical outflow pathways [30].

A couple of weaknesses of our study should be addressed. Although the left BG infarct was associated with significant cognitive impairment and micrographia in our patient, we cannot exclude the possibility that lesions in both basal ganglia were required to generate these findings since our patient also had a chronic right BG infarct. Another weakness is that it is possible that, due to the resolution of our patient's MRI, involvement of the anterior portion of the thalamus may not have been definitively excluded. As noted in the literature, various and significant cognitive changes including apathy, word-finding difficulties, perseveration, superposition of unrelated information, and memory problems can also occur after thalamic strokes [31-33].

The literature is conflicting on whether cognitive effects are associated with GP lesions. However, after suffering a GP infarct, our patient demonstrated major cognitive deficits, particularly in the domains of short-term and working memory, visuospatial ability, executive function, attention, and concentration. Our patient also displayed evidence of micrographia, though we were unable to further evaluate this finding because the patient was lost to follow up. BG region strokes appear to be associated with micrographia, albeit infrequently. Our case emphasizes that GP lesions can cause significant cognitive sequelae and likely adds to our understanding on the localizations of micrographia. Neuropsychological testing should be considered in patients suffering from GP strokes.

\section{Conflict of Interests}

The authors declare that they have no conflict of interests.

\section{Authors' Contribution}

Sarah Nelson collected the data and drafted and edited the paper. Hassanain Toma conceptualized the study and edited the paper. Haley LaMonica and Tinatin Chabrashvili supervised the study and edited the paper.

\section{References}

[1] K. P. Bhatia and C. D. Marsden, "The behavioural and motor consequences of focal lesions of the basal ganglia in man," Brain, vol. 117, no. 4, pp. 859-876, 1994.

[2] A. Münchau, D. Mathen, T. Cox, N. P. Quinn, C. D. Marsden, and K. P. Bhatia, "Unilateral lesions of the globus pallidus: report of four patients presenting with focal or segmental dystonia," Journal of Neurology, Neurosurgery \& Psychiatry, vol. 69, no. 4, pp. 494-498, 2000.

[3] J. Ghika, F. Ghika-Schmid, H. Fankhauser et al., "Bilateral contemporaneous posteroventral pallidotomy for the treatment of Parkinson's disease: Neuropsychological and neurological side effects: report of four cases and review of the literature," Journal of Neurosurgery, vol. 91, no. 2, pp. 313-321, 1999.

[4] C. Junque, M. Alegret, F. A. Nobbe et al., "Cognitive and behavioral changes after unilateral posteroventral pallidotomy : relationship with lesional data from MRI," Movement Disorders, vol. 14 , no. 5, pp. 780-789, 1999.

[5] W. J. Lombardi, R. E. Gross, L. L. Trepanier, A. E. Lang, A. M. Lozano, and J. A. Saint-Cyr, "Relationship of lesion location to cognitive outcome following microelectrode-guided pallidotomy for Parkinson's disease. Support for the existence of cognitive circuits in the human pallidum," Brain, vol. 123, no. 4, pp. 746-758, 2000.

[6] C. Saleh and M. S. Okun, "A clinical review of deep brain stimulation and its effects on limbic basal ganglia circuitry," Frontiers in Bioscience, vol. 13, no. 15, pp. 5708-5731, 2008.

[7] Z. S. Nasreddine, N. A. Phillips, V. Bédirian et al., "The Montreal Cognitive Assessment, MoCA: a brief screening tool for mild cognitive impairment," Journal of the American Geriatrics Society, vol. 53, no. 4, pp. 695-699, 2005.

[8] M. Giroud, M. Lemesle, G. Madinier, T. Billiar, and R. Dumas, "Unilateral lenticular infarcts: radiological and clinical syndromes, aetiology, and prognosis," Journal of Neurology Neurosurgery and Psychiatry, vol. 63, no. 5, pp. 611-615, 1997.

[9] S. H. Kim, K. H. Park, Y. H. Sung, Y. B. Lee, H. M. Park, and D. J. Shin, "Dementia mimicking a sudden cognitive and behavioral change induced by left globus pallidus infarction: review of two cases," Journal of the Neurological Sciences, vol. 272, no. 1-2, pp. 178-182, 2008.

[10] E. Mori, "Impact of subcortical ischemic lesions on behavior and cognition," Annals of the New York Academy of Sciences, vol. 977, pp. 141-148, 2002.

[11] B. Pillon, C. Ardouin, K. Dujardin et al., "Preservation of cognitive function in dystonia treated by pallidal stimulation," Neurology, vol. 66, no. 10, pp. 1556-1558, 2006.

[12] A. Fasano, P. Mazzone, C. Piano, D. Quaranta, F. Soleti, and A. R. Bentivoglio, "GPi-DBS in Huntington's disease: results on motor function and cognition in a 72-year-old case," Movement Disorders, vol. 23, no. 9, pp. 1289-1293, 2008.

[13] G. Vingerhoets, C. Van Der Linden, E. Lannoo et al., "Cognitive outcome after unilateral pallidal stimulation in Parkinson's disease," Journal of Neurology Neurosurgery and Psychiatry, vol. 66 , no. 3, pp. 297-304, 1999.

[14] M. S. Okun, H. H. Fernandez, S. S. Wu et al., "Cognition and mood in Parkinson's disease in subthalamic nucleus versus globus pallidus interna deep brain stimulation: the COMPARE trial," Annals of Neurology, vol. 65, no. 5, pp. 586-595, 2009. 
[15] M. Jahanshahi, C. M. Ardouin, R. G. Brown et al., "The impact of deep brain stimulation on executive function in Parkinson's disease," Brain, vol. 123, no. 6, pp. 1142-1154, 2000.

[16] A. I. Tröster, J. A. Fields, S. B. Wilkinson et al., "Unilateral pallidal stimulation for Parkinson's disease: neurobehavioral functioning before and 3 months after electrode implantation," Neurology, vol. 49, no. 4, pp. 1078-1083, 1997.

[17] D. Mastennan, A. DeSalles, R. W. Baloh et al., "Motor, cognitive, and behavioral performance following unilateral ventroposterior pallidotomy for Parkinson disease," Archives of Neurology, vol. 55, no. 9, pp. 1201-1208, 1998.

[18] G. J. Demakis, M. G. Mercury, J. J. Sweet, M. Rezak, T. Eller, and S. Vergenz, "Motor and cognitive sequelae of unilateral pallidotomy in intractable Parkinson's disease: electronic measurement of motor steadiness is a useful outcome measure," Journal of Clinical and Experimental Neuropsychology, vol. 24, no. 5, pp. 655-663, 2002.

[19] R. Scott, R. Gregory, N. Hines et al., "Neuropsychological, neurological and functional outcome following pallidotomy for Parkinson's disease: a consecutive series of eight simultaneous bilateral and twelve unilateral procedures," Brain, vol. 121, no. 4, pp. 659-675, 1998.

[20] A. Gironell, J. Kulisevsky, L. Rami, N. Fortuny, C. GarcíaSánchez, and B. Pascual-Sedano, "Effects of pallidotomy and bilateral subthalamic stimulation on cognitive function in Parkinson disease: a controlled comparative study," Journal of Neurology, vol. 250, no. 8, pp. 917-923, 2003.

[21] B. Schmand, R. M. A. De Bie, M. Koning-Haanstra, J. S. De Smet, J. D. Speelman, and A. H. Van Zomeren, "Unilateral pallidotomy in PD: a controlled study of cognitive and behavioral effects," Neurology, vol. 54, no. 5, pp. 1058-1064, 2000.

[22] L. A. Klatka, M. H. Depper, and A. M. Marini, "Infarction in the territory of the anterior cerebral artery," Neurology, vol. 51, no. 2, pp. 620-622, 1998.

[23] K. Maeda, R. Idehara, and T. Shiraishi, "Micrographia and abulia due to frontal subcortical infarction," Internal Medicine, vol. 51, no. 14, pp. 1953-1954, 2012.

[24] K. Maeda, A. Nakajima, N. Ogawa, and H. Kawai, "Micrographia associated with motor neglect," Internal Medicine, vol. 50, no. 8, p. 943, 2011.

[25] A. K. Troyer, S. E. Black, M. L. Armilio, and M. Moscovitch, "Cognitive and motor functioning in a patient with selective infarction of the left basal ganglia: evidence for decreased nonroutine response selection and performance," Neuropsychologia, vol. 42, no. 7, pp. 902-911, 2004.

[26] J. S. Kim, J. H. Im, S. U. Kwon, J. H. Kang, and M. C. Lee, "Micrographia after thalamo-mesencephalic infarction: evidence of striatal dopaminergic hypofunction," Neurology, vol. 51, no. 2, pp. 625-627, 1998.

[27] F. Capone, F. Pilato, P. Profice, E. Pravatà, and V. di Lazzaro, "Neurological picture. A case of stroke induced micrographia," Journal of Neurology, Neurosurgery, and Psychiatry, vol. 80, no. 12, p. 1356, 2009.

[28] M. A. Marinella, "Subcortical stroke presenting as micrographia," The American Journal of Emergency Medicine, vol. 25, no. 1, pp. 89-90, 2007.

[29] P. Derkinderen, S. Dupont, J. S. Vidal, F. Chedru, and M. Vidailhet, "Micrographia secondary to lenticular lesions," Movement Disorders, vol. 17, no. 4, pp. 835-837, 2002.

[30] C. Blahak, H.-H. Capelle, H. Baezner, T. M. Kinfe, M. G. Hennerici, and J. K. Krauss, "Micrographia induced by pallidal
DBS for segmental dystonia: a subtle sign of hypokinesia?" Journal of Neural Transmission, vol. 118, no. 4, pp. 549-553, 2011.

[31] F. Ghika-Schmid and J. Bogousslavsky, "The acute behavioral syndrome of anterior thalamic infarction: a prospective study of 12 cases," Annals of Neurology, vol. 48, no. 2, pp. 220-227, 2000.

[32] E. Carrera and J. Bogousslavsky, "The thalamus and behavior: effects of anatomically distinct strokes," Neurology, vol. 66, no. 12, pp. 1817-1823, 2006.

[33] Y. Nishio, M. Hashimoto, K. Ishii, and E. Mori, "Neuroanatomy of a neurobehavioral disturbance in the left anterior thalamic infarction," Journal of Neurology, Neurosurgery and Psychiatry, vol. 82, no. 11, pp. 1195-1200, 2011. 


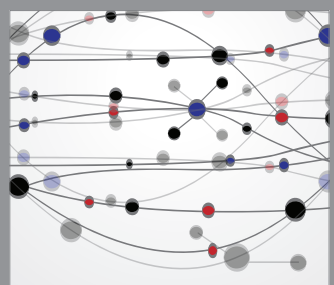

The Scientific World Journal
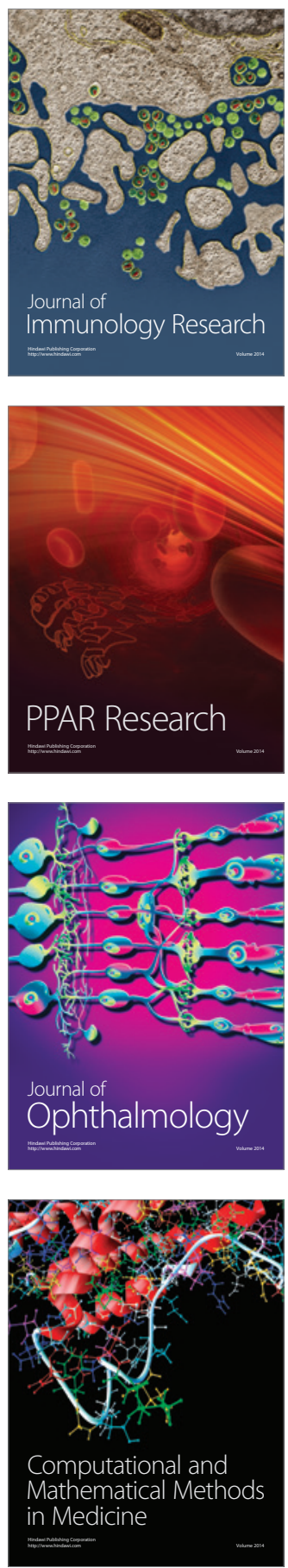

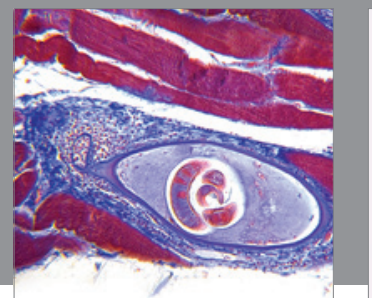

Gastroenterology

Research and Practice
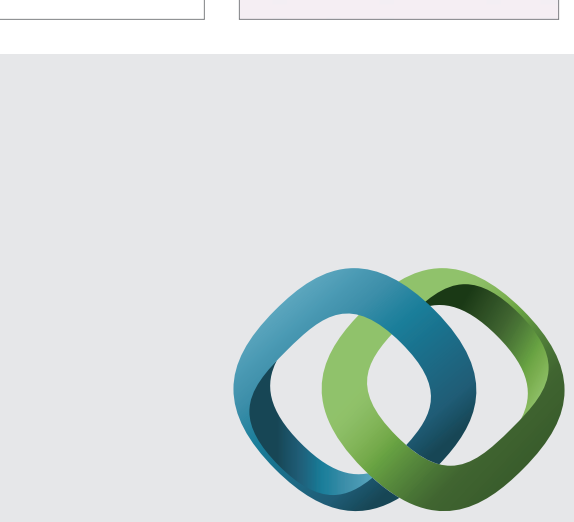

\section{Hindawi}

Submit your manuscripts at

http://www.hindawi.com
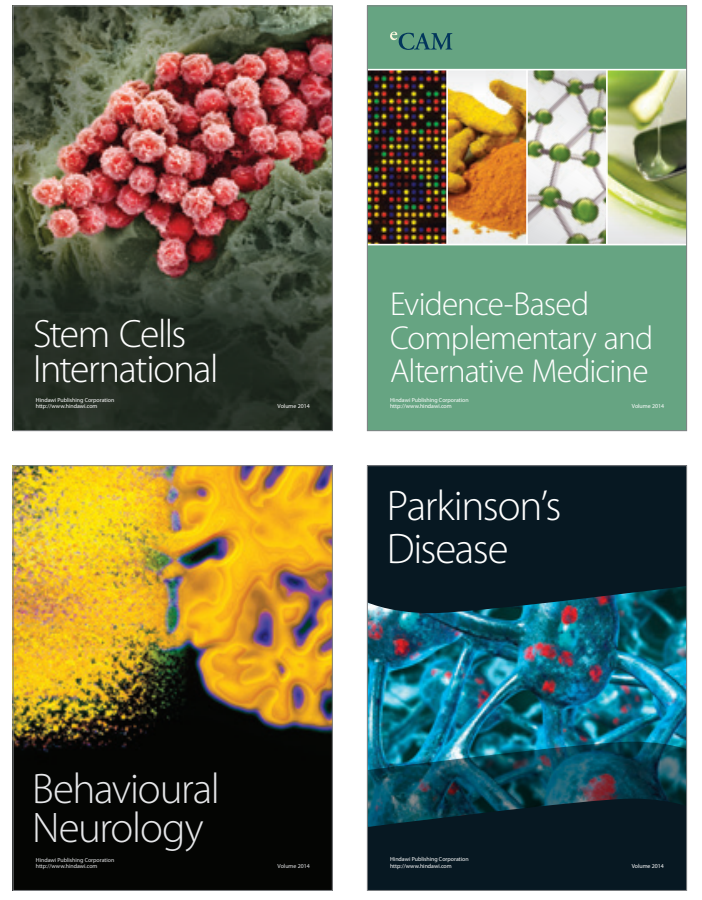
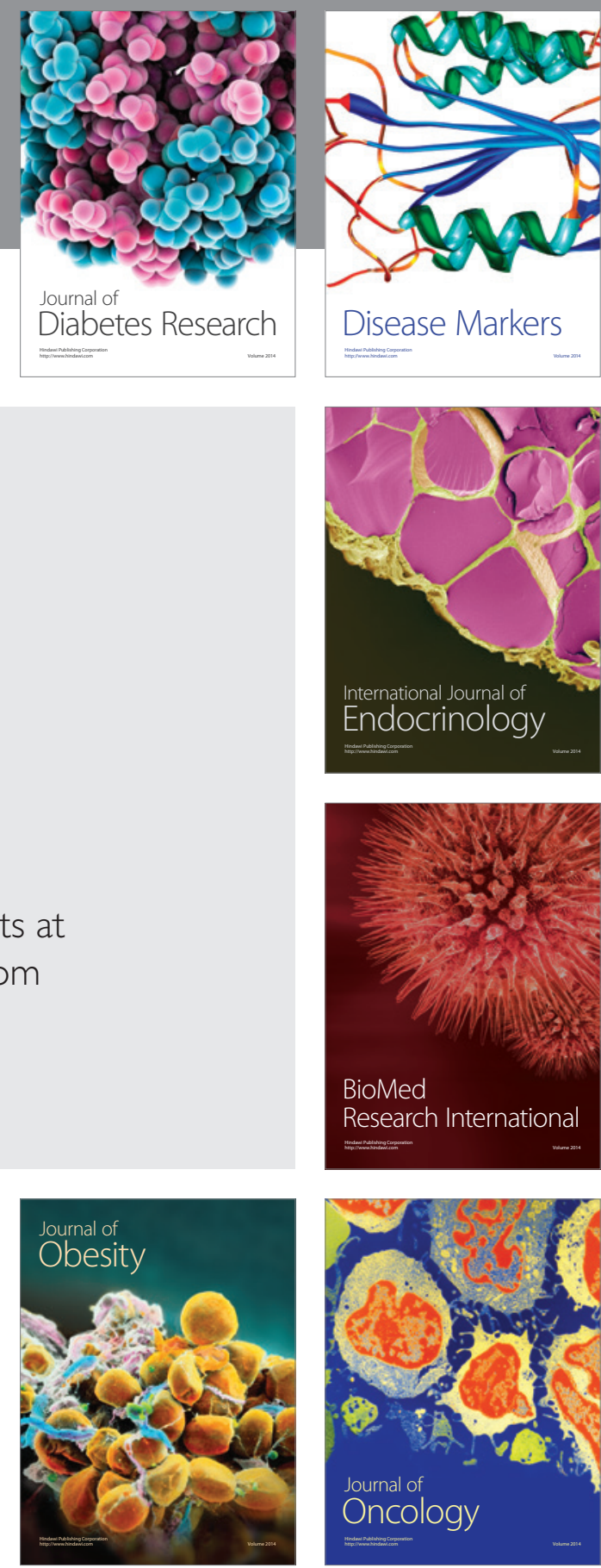

Disease Markers
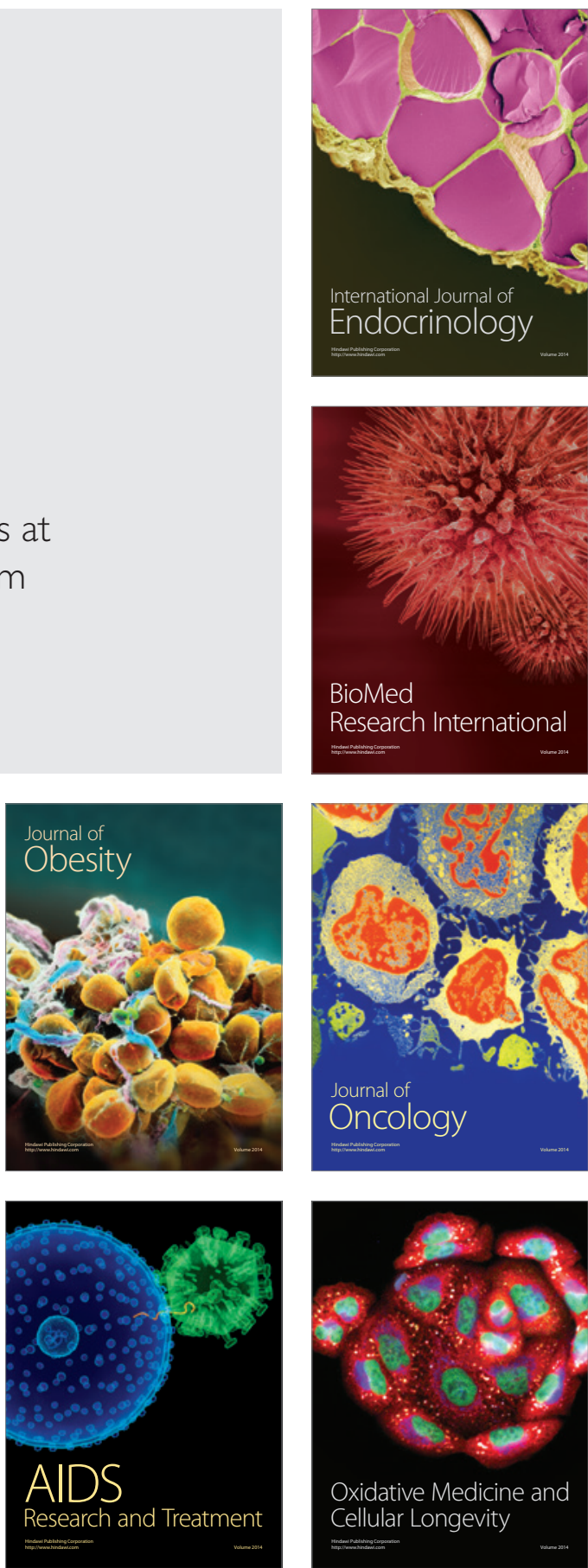